\title{
Análisis factorial del componente de gestión del nuevo índice de medición municipal en Colombia
}

\author{
Vargas Ramírez, Iván Rodrigo; Zambrano Valdivieso, Óscar Javier \\ Análisis factorial del componente de gestión del nuevo índice de medición municipal en Colombia \\ Revista Economía y Política, núm. 31, 2020 \\ Universidad de Cuenca, Ecuador \\ Disponible en: http://www.redalyc.org/articulo.oa?id=571162102007 \\ DOI: https://doi.org/10.25097/rep.n31.2020.05 \\ La Universidad de Cuenca en Ecuador, conserva los derechos patrimoniales (copyright) de las obras publicadas, \\ y favorece y permite la reutilización de las mismas bajo la licencia Creative Commons Atribución-NoComercial- \\ Compartirlgual 4.0 Internacional (CC BY-NC-SA 4.0), por lo cual se pueden copiar, usar, difundir, transmitir y \\ exponer públicamente, siempre que: a. Se cite la autoría y fuente original de su publicación (revista, editorial, \\ URL y DOI de la obra). b. No se usen para fines comerciales u onerosos. c. Se mencione la existencia y \\ especificaciones de esta licencia de uso. \\ Esta obra está bajo una Licencia Creative Commons Atribución-NoComercial-Compartirlgual 4.0 Internacional.
}


Artículos

\section{Análisis factorial del componente de gestión del nuevo índice de medición municipal en Colombia}

Factorial analysis of the management component of the new municipal measurement index in Colombia

Iván Rodrigo Vargas Ramírez

COLCIENCIAS, Colombia

DOI: https://doi.org/10.25097/rep.n31.2020.05

msc.ivanvargas@gmail.com

Redalyc: http://www.redalyc.org/articulo.oa?

Óscar Javier Zambrano Valdivieso

Corporación Universitaria Minuto de Dios

UNIMINUTO, Colombia

ozambranov1@uniminuto.edu.co

Recepción: 26 Septiembre 2019

Aprobación: 03 Enero 2020

\section{Resumen:}

La planeación es un proceso estratégico para la consecución de resultados al interior de las instituciones. En la dirección pública, las entidades territoriales tienen el derecho y la obligación de organizar y desarrollar una serie de estrategias que les permitan alcanzar mayores niveles de calidad de vida para sus comunidades. Con base en un estudio cuantitativo se desarrolla un diseño de experimentos con cuatro factores que miden el componente de la gestión realizada en 1121 municipios del país en el 2017; entendiendo las oportunidades y orientaciones que requieren los alcaldes para mejorar el índice de desempeño municipal, mediante análisis factorial del componente de gestión del nuevo índice de medición municipal en Colombia. El análisis factorial es una técnica multivariada que permite reducir el "tamaño" de un problema sin "demasiada pérdida de información”, es decir, este análisis (análisis de factores comunes o componentes principales) procura reducir la data que nos suministra una matriz de correlaciones para hacerla más expeditamente interpretable. Se procura descubrir una contestación a la interrogante ¿Por qué unas variables se relacionan más entre sí y menos con otras?. La información utilizada es fuente del Departamento Nacional de Planeación (DANE) y fundamentado en el estudio multivariado se concluye que las alcaldías requieren trabajar principalmente en ejecutar recursos, en el ordenamiento territorial, así como en el gobierno abierto y transparente.

Palabras ClaVe: Desempeño administrativo, municipio, organización, entidad territorial, gestión, resultados.

\section{ABstract:}

Planning is a strategic process for achieving results within institutions. At the public level, territorial entities have the right and the obligation to organize and develop a series of strategies that allow them to achieve higher levels of quality of life for their communities. Based on a quantitative study, a design of experiments is developed with four factors that measure the management component carried out in 1121 municipalities of the country in 2017; understanding the opportunities and orientations required by mayors to improve the municipal performance index, through factor analysis of the management component of the new municipal measurement index in Colombia. The factorial analysis is a multivariate technique that allows to reduce the "size" of a problem without "too much loss of information", that is to say, this analysis (analysis of common factors or main components) tries to reduce the data that provides us with a correlation matrix to make it more expeditiously interpretable. It seeks to discover an answer to the question Why do some variables relate more to each other and less to others? The information used is a source of the National Planning Department (DANE) and based on the multivariate study, it is concluded that the municipalities need to work mainly on the execution of resources, on territorial planning, as well as on open and transparent government.

KEYWORDS: Administrative performance, municipality, organization, territorial entity, management, results.

\section{INTRODUCCIÓN}

La modernización y transformación de la administración pública es un eje central de la actual agenda política de los países latinoamericanos (CLAD, 1999). Para Sen (2017), estos procesos son desarrollados mediante 
el adecuado uso de los recursos del Estado que maximicen el bienestar social en un territorio determinado. Este fenómeno ha sido producto de la globalización y del relacionamiento entre sectores, países, gremios y comunidades que crean nuevos escenarios de gestión pública (Organización para la Cooperación y Desarrollo Económico, 2010).

Colombia, desde inicios de la década de los noventa, está desarrollando grandes procesos de innovación en el diseño y la implementación de políticas públicas que buscan el cumplimiento de los objetivos, metas, programas y proyectos diseñados en la planeación del Estado, los cuales están orientados por los principios del enfoque de la Nueva Gestión Pública promulgada por el CLAD y la OCDE respectivamente (Pollit \& Bouckaert, 2004; Prats, 2004; Christensen \& Lagreid, 2010).

Para Turnley, Bolino, Lester \& Bloodgood (2003), las organizaciones estatales se desarrollan en ambientes caracterizados por altos niveles de competitividad, incertidumbre y de avances tecnológicos que permiten el uso de los datos públicos como sistemas de información para el mejoramiento en la toma de decisiones desde la perspectiva nacional con enfoque territorial. Por otra parte, Topa \& Palací (2004), concluyen que estos cambios en el sector se evidencian en la reestructuración de la jerarquía y la cultura organizacional, creando mayores niveles de flexibilidad interna y mejores respuestas ante los estímulos externos.

En estos nuevos escenarios, Seligman, Steen, Park \& Peterson (2005) y Vargas (2019), determinan que la gestión de la felicidad en el trabajo, la estructuración de sistemas de medición del desempeño de lo público y la eficiencia en el uso de los recursos se convierten en escenarios estratégicos para impulsar la efectividad de estas organizaciones y el mejoramiento de la calidad de vida de las personas.

Estos avances en el país surgen como el resultado alcanzado en la psicología organizacional positiva, el funcionamiento ideal en la salud y la bienandanza de los colaboradores en entornos determinados (Carretero, 2017). Sin embargo, Fisher \& Locke (1992) argumentan que existe poca literatura respecto a los nuevos conceptos, métodos e instrumentos implementados en la administración pública que permitan el análisis y la evaluación de los mismos en las realidades de las comunidades.

Los municipios, como entidades territoriales en Colombia, son constitucionalmente encargados de la administración y gestión de los recursos del Estado para solucionar y responder a las necesidades de las comunidades. Para ello, utilizan los planes de desarrollo como principal instrumento de planificación de estrategias, resultados y recursos que deben gestionar en su periodo de mandato. El Departamento Nacional de Planeación con base en lo expuesto anteriormente, ha diseñado un nuevo Indicador de Medición del Desempeño de los Municipios del país que anualmente mide los componentes de gestión y de resultados alcanzados por los Alcaldes a través de ocho dimensiones, cada una de ellas valorada entre 0 como mínimo y 100 como máximo alcanzable.

Como lo argumentan Avella \& Oliva (2010), existen diversas formas de medir una variable latente y una de ellas es la arquitectura de índices sintéticos, que sintetizan la data de las variables cualitativas y/o cuantitativas; es destacable que con la miscelánea de los índices simples, se edifican índices sintéticos que no expresan todos los componentes que refieren la variable latente, más bien, sólo son una proximidad a ella.

El índice sintético es una medida derivada de indicadores simples, mediante una suma ponderada de estos indicadores; se evidencia igualmente, que concurren muchas formas de computar las ponderaciones, como: métodos estadísticos, cálculos matemáticos y heurísticos.

Para la presente investigación, se determinó que el Análisis Factorial seria el método para analizar un Índice Sintético idóneo para la medición de la gestión municipal en Colombia; Ferrando \& Anguiano-Carrasco (2010), confirman que el análisis factorial (AF) se ha perfeccionado extensamente a lo largo de sus más de 100 años de presencia.

Spearman (1904), fue quien expuso este modelo para certificar su teoría de la inteligencia, el cual ha dado lugar a una extensa estirpe de modelos que se emplean en diversos escenarios.

$\mathrm{El} \mathrm{AF}$ es un modelo estadístico que representa las relaciones entre un conjunto de variables; plantea que estas relaciones pueden explicarse a partir de una serie de variables no observables (latentes) denominadas 
factores, siendo el número de factores substancialmente menor que el de variables. El modelo se obtiene directamente como extensión de algunas de las ideas básicas de los modelos de regresión lineal y de correlación parcial. Del primer modelo se derivan las ecuaciones fundamentales del AF; del segundo se derivan las ideas clave para evaluar el ajuste del modelo a los datos.

Por lo anterior, la presente investigación estudia el componente de gestión del indicador con sus cuatro dimensiones (movilidad de recursos propios, ejecución de recursos, gobierno abierto y transparente, y ordenamiento territorial) a través del análisis factorial, como un ejercicio cuantitativo que contribuya a la interpretación de los resultados obtenidos en la vigencia 2016 por todas las alcaldías del país, generando una serie de recomendaciones que incentiven los procesos de gestión del quehacer público en el territorio y con proyección nacional y regional.

\section{Administración}

La administración es una disciplina científica que estudia el comportamiento de la organización (Agüero, 2007), su enfoque principal es la planeación, la implementación y la evaluación de los recursos necesarios para el cumplimiento de sus objetivos (Dubrin, 2000).

Dentro de los principales aportes en la materia, Taylor (1911), inicia estableciendo que la administración es concebida bajo el carácter científico y racional del trabajo en términos de tiempos, movimientos y costos que buscan la optimización de los resultados y el aumento de las utilidades.

Posteriormente, Fayol (1918) entiende la administración como la búsqueda de la eficiencia de la organización bajo un enfoque gerencial, en donde el operario y el directivo son seres humanos, que tienen unas expectativas, necesidades y requerimientos que en la organización se pueden alcanzar; mientras que Gulick (1937) enfatiza en el liderazgo como el elemento principal para el rendimiento organizacional, que a través del proceso de toma de decisiones garantiza el cumplimiento de los objetivos determinados.

Por otra parte, Drucker (1966) argumenta que la productividad es el verdadero objetivo de la administración, porque se concentra en generar instrumentos y metodologías que busquen la eficiencia y efectividad de la gestión y por último, Gibson (1997) y Toffer (1997) presentan como el gran reto de la administración a la competitividad, que es aquella que permite la generación de ventajas competitivas mediante el buen uso del conocimiento y la aplicación de metodologías integrales y ágiles en las organizaciones.

\section{Administración pública}

Weber (1922) conceptualiza la administración del Estado desde la perspectiva de la burocracia, entendida como el desarrollo de actividades lógicas y organizadas realizadas para mejorar la eficiencia en los resultados de interés social. En concordancia, Thompson (2008) sintetiza que la administración pública está orientada directamente con las organizaciones públicas cuyo objetivo es el bienestar social, y la consolidación del carácter preventivo y proteccionista de los marcos jurídicos constitucionales (Sánchez, 2009; Claver, et al. 1999). Sin embargo, Luhmann (1971), concluye que la administración pública en los territorios alcanza su mayor impacto cuando es acordada y priorizada por las necesidades de la comunidad, lo que para Bueno (1993) se denomina el poder negociador y decisional del Estado y sus directivos

\section{Nueva Gestión Pública}

En la actualidad, la gestión pública ha sido abordada por nuevas tendencias originadas en el sector privado y a partir de los casos de éxito del sector público logrado en países anglosajones, y ha modificado la estructura y entendimiento de la función pública, al punto de que según Dunleavy \& Hood (1994) en la función pública, se establecen principios, metodologías, prácticas e instrumentos que buscan las mejores respuestas del Estado 
frente las necesidades y expectativas de las personas y comunidades; como por ejemplo, la flexibilización de las organizaciones rígidas y verticales del sector público, el uso de los sistemas y plataformas de información que faciliten la recolección, almacenamiento, reporte y toma de decisiones, la innovación abierta, la medición del desempeño de la gestión pública, la gestión por resultados, la orientación de cliente al ciudadano, requieren de una satisfacción, de una participación en la agenda y una evaluación de los productos y recursos del Estado.

\section{Entidades territoriales}

La Constitución Política de Colombia de 1991, establece en su artículo primero, que el país está conformado por entidades territoriales autónomas alienadas con el gobierno central (Manrique, 2006). Una entidad territorial es una persona jurídica de derecho público que goza de autonomía para la administración de sus necesidades, expectativas e intereses con base en el marco constitucional. El artículo 287 de la Constitución determina que podrán gobernarse de forma propia, liderar las competencias impuestas por la Ley, gestionar recursos y participar en las rentas del país. Ejemplo de ellas son: los departamentos, municipios, resguardos indígenas, provincias y distritos, entre otros. En cada una de ellos, existe una autoridad público administrativa de elección popular encargada de dirigirla por un periodo de 4 años. En esta investigación los Alcaldes, son los encargados de coordinar los municipios del país, desarrollando la formulación, implementación y evaluación de los planes de desarrollo que administren los recursos del territorio para el bienestar social (Código de Régimen Departamental, 1986; Cedamanos, 2016; Villegas, 2007).

\section{Desempeño administrativo en los municipios de Colombia}

Para Cheng (2006), el desempeño administrativo es el cumplimiento de los objetivos planteados en una entidad territorial, su evaluación es un principio de la Nueva Gestión Pública. Murphy \& Williams (1995) señalan que la comunidad y las instituciones requieren conocer y participar en el avance de la gestión de sus administraciones. Para el caso colombiano, el desempeño administrativo de las alcaldías se evalúa por medio del Departamento Nacional de Planeación (2017), quien utiliza el Nuevo Indicador de Medición del Desempeño Municipal que está compuesto por los componentes de gestión y resultados, cada uno de ellos aporta un 50\% del valor final, como se presenta en la Figura 1.

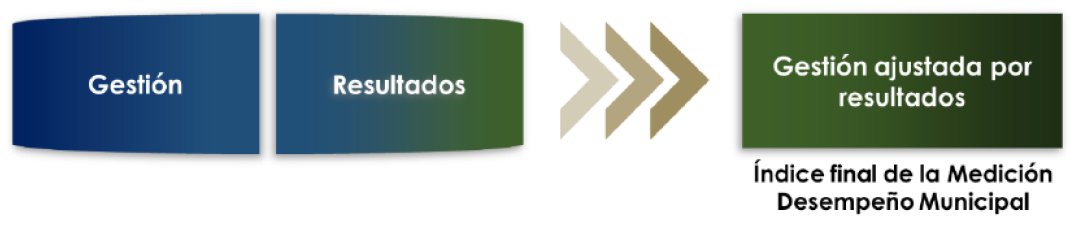

FIGURA 1.

Estructura de nueva medición del desempeño municipal

DNP (2017)

El componente de gestión se define como las decisiones y acciones que perfecciona la administración pública para la consecución y movilización de los insumos del sector público, que están orientados a la mayor prosperidad de la urbe y el desarrollo social; la gestión está determinada por las cuatro dimensiones representadas en la Tabla 1, que a través de variables internas definen el nivel de cada una de las entidades territoriales por cada vigencia ${ }^{[1]}$, por lo que entender cómo se relacionan entre ellas, con respecto al total del índice alcanzado por los Alcaldes del país, permitirá generar unas líneas de acción claras en pro a la optimización del componente de gestión y el de resultados. 
Entender esta organización de las cuatro dimensiones de gestión fortalecerá el esquema y la ejecución de políticas públicas que promuevan la equidad y prosperidad de sus habitantes.

TABLA 1.

\section{Dimensiones del componente gestión}

\begin{tabular}{|c|c|}
\hline Dimensiones & Descripción \\
\hline 1.Movilización de recursos & $\begin{array}{l}\text { Mide los ingresos tributarios y no tributarios per cápita } \\
\text { recaudados tanto por los instrumentos de } \\
\text { ordenamiento territorial como por otras fuentes. Se } \\
\text { alimenta de Ingresos tributarios y no tributarios per } \\
\text { cápita, recaudo por instrumentos de organización } \\
\text { territorial y participación de los recursos propios de } \\
\text { inversión. }\end{array}$ \\
\hline 2.Ejecución de recursos & $\begin{array}{l}\text { Se centra en el efectivo uso de los recursos que una } \\
\text { entidad territorial tiene para trabajar en la solución de } \\
\text { necesidades de una comunidad. Se nutre de las } \\
\text { variables de utilización de recursos del sistema general } \\
\text { de participaciones, recursos propios, sistema general de } \\
\text { regalias y otras fuentes de financiación. }\end{array}$ \\
\hline $\begin{array}{l}\text { 3.Gobierno abierto y } \\
\text { transparente }\end{array}$ & $\begin{array}{l}\text { Es la organización de la información interna de las } \\
\text { entidades territoriales y la capacidad de difundirla } \\
\text { tanto por oferta como por demanda Se constituye a } \\
\text { través de las variables de organización de la } \\
\text { información, rendición de cuentas y atención al } \\
\text { ciudadano. }\end{array}$ \\
\hline 4.Ordenamiento territorial & $\begin{array}{l}\text { Es la capacidad para recaudar efectivamente el predial } \\
\text { del municipio se basa en las variables de uso de } \\
\text { instrumentos de recaudo y el recaudo efectivo predial. }\end{array}$ \\
\hline
\end{tabular}

Elaboración propia de los autores (2018)

El segundo componente del indicador de la nueva medición del desempeño municipal es el de resultados, el cual busca minimizar el gap socio económico a lo largo del territorio. Para lograrlo se establece la valoración de resultados en cuatro categorías; salud, educación, acceso a servicios y seguridad relacionados con las variables que la determinan en la Tabla 2. 
Iván Rodrigo Vargas Ramírez, et aL. AnÁlisis factorial del componente de gestión del nUevo índice ...

TABLA 2.

Categorías del componente de resultados

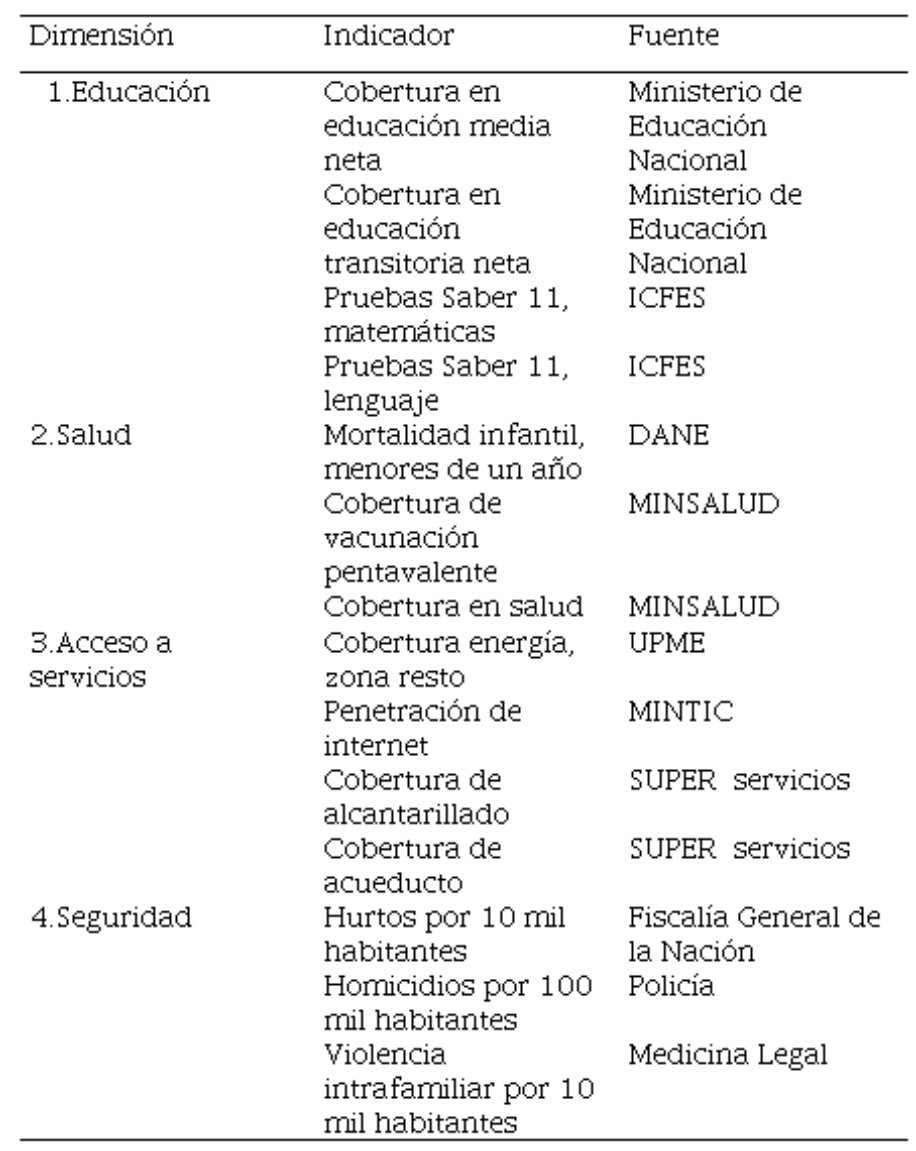

Elaboración propia de los autores (2018)

Con base en lo anterior, el nuevo indicador por cada alcaldía resulta de la suma aritmética de los dos componentes definidos. Cada componente, a su vez, tiene una serie de dimensiones, las cuales están compuestas por variables que obtienen la información de fuente primaria directa de las entidades territoriales o de fuente secundaria de organizaciones estatales del orden nacional. A continuación, se presentan las ponderaciones de cada componente. 


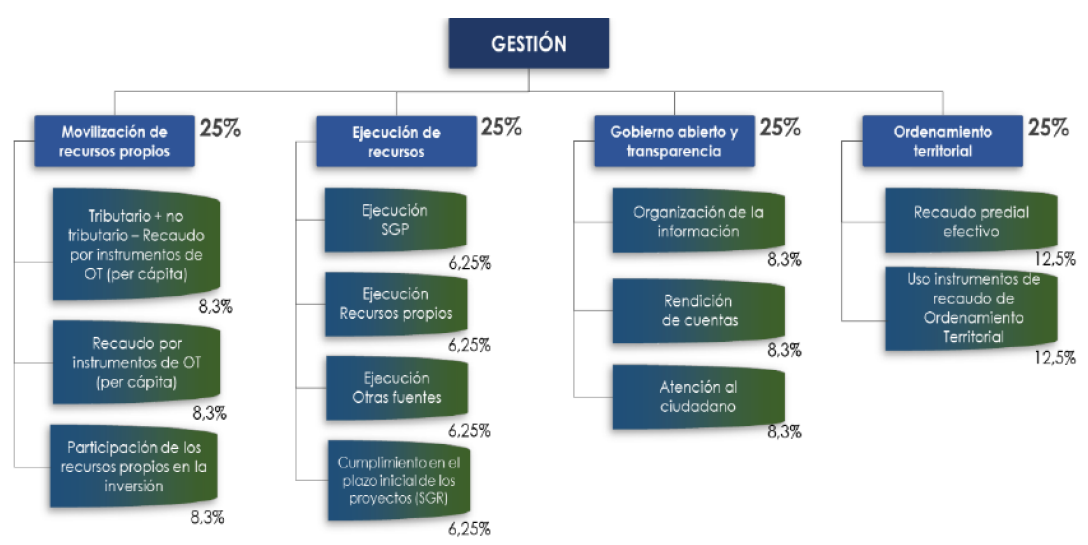

FIGURA 2.

Ponderación de las categorías y variables de los componentes del nuevo índice Elaboración propia de los autores (2018) con base en DNP (2017)

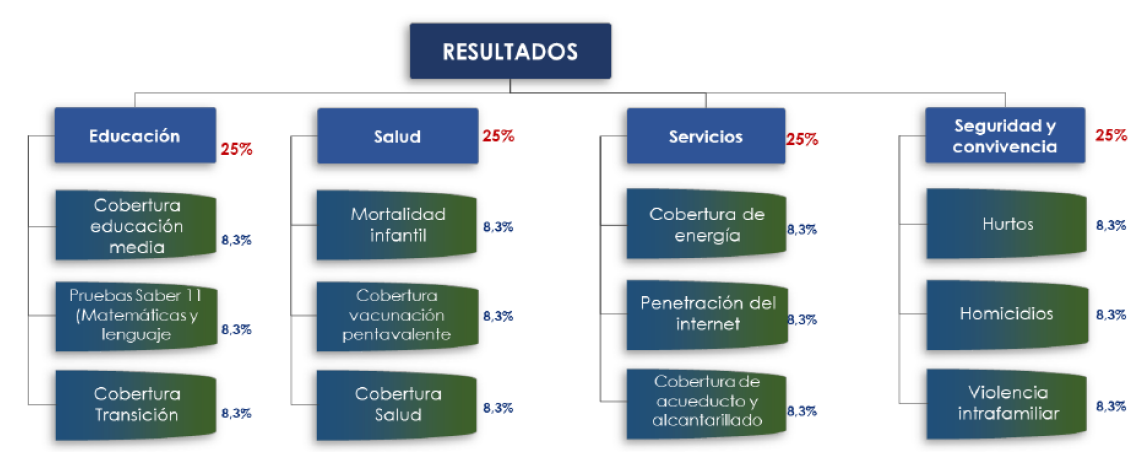

FIGURA 3.

Ponderación de las categorías y variables de los componentes del nuevo índice Elaboración propia de los autores (2018) con base en DNP (2017)

\section{Análisis factorial}

García, Enrique, \& Tello (2011) demostraron que, aunque se considera a Galton (1883) el pionero en la técnica del análisis factorial, fueron dos discípulos suyos Pearson y Spearman, quienes forjaron los cimientos del análisis factorial cuando pretendieron definir y medir objetivamente la inteligencia. Spearman (1904) publicó en el American Journal of Psychology un artículo titulado «General Intelligence Objetively Determined and Measured», en el que confirma que era suficiente un solo factor, una sola variable hipotética, para exponer las intercorrelaciones de un agregado de tests cognoscitivos, a esta variable la nombró "factor general de inteligencia, el factor $« \mathrm{~g} » "$.

Realmente parece que esta hipótesis era plausible cuando se utilizaban las matrices de intercorrelaciones obtenidas a partir de los test que había utilizado Spearman, pero cuando se consideraron otras matrices de correlaciones obtenidas a partir de otros test, se vio que la hipótesis de Spearman era bastante simplicista y fueron apareciendo otras hipótesis basadas en factores múltiples. (García, Enrique, \& Tello, 2011).

L. L. Thurstone en América y C. Burt y G. H. Thomson en Gran Bretaña (Thurstone, 1931; 1935; 1947; Burt, 1949; Thomson, 1951), aparecen como precursores de este nuevo método.

Garmendia (2007), en su investigación, confirma que el análisis factorial es una técnica de análisis multivariante que se emplea tanto para revelar y estudiar la disposición en las relaciones entre un conjunto de 
variables como para comprimir el número de variables. Toma las correlaciones entre variables para afirmar que no son aleatorias, sino que corresponden a la presencia subyacente de componentes usuales entre ellas.

Existen constructos, tales como: el apoyo social, la satisfacción o la calidad de vida que se requieren analizar, pero que no se logran medir con una sola pregunta debido a que son fenómenos complejos como lo argumentan González e Ibáñez (2001) y se convierten entonces, en el resultado de la medición de un conjunto de características. En palabras de Morrison (1990), el análisis factorial consiente en revelar patrones escuetos en el patrón de relaciones entre variables; indaga si las variables observadas logran ser expresadas en términos de un menor guarismo de variables llamadas factores. Las variables observadas, por lo tanto, consiguen ser modeladas como combinaciones lineales de factores más el error de medida.

El análisis factorial se clasifica en dos tipos; confirmatorio, el cual permite comprobar hipótesis, es decir, busca determinar si los factores y las variables que los conforman concuerdan con una teoría preestablecida y exploratorio, esto es, permite generar hipótesis sobre las variables subyacentes a los datos, por lo que a priori, cualquier variable puede estar asociada a cualquier factor.

\section{Metodología}

El presente estudio se desarrolla con un enfoque cuantitativo basado en una revisión documental de 22 artículos indexados, identificados por las palabras claves de administración pública y territorial, además utiliza el análisis factorial bajo el diseño de experimentos factoriales de tipo $2^{\mathrm{k}=4}$ para la generación, análisis e interpretación de la información reportada por las alcaldías del país ante el Departamento Nacional de Planeación. En la Tabla 3 se aprecia el diseño metodológico y las variables utilizadas:

TABLA 3.

Diseño metodológico

\begin{tabular}{|c|c|}
\hline Ítem & Etapa \\
\hline 1 & $\begin{array}{l}\text { Identificación de } 4 \text { categorias con sus dos } \\
\text { valores posibles del componente de gestión } \\
\text { del indice de medición. }\end{array}$ \\
\hline 2 & $\begin{array}{l}\text { Construcción de las tablas de referencia por } \\
\text { categorias, experimentos en } 16 \\
\text { posibilidades con base en } 4 \text { categorias. } \\
\text { (Calculando las veces que se repite cada } \\
\text { factor en la base de datos y el total por cada } \\
\text { escenario posible) }\end{array}$ \\
\hline 3 & $\begin{array}{l}\text { Establecimiento de la suma de cuadrados, } \\
\text { grados de libertad y los cuadrados medios } \\
\text { para cada uno de los } 16 \text { escenarios. }\end{array}$ \\
\hline 4 & $\begin{array}{l}\text { Determinación del residual con base en los } \\
\text { grupos de datos seleccionados, del f ratio y } \\
\text { f tabla de cada factor y su posible } \\
\text { combinación, definiendo los significativos y } \\
\text { los que no lo son para el ejercicio. }\end{array}$ \\
\hline 5 & $\begin{array}{l}\text { Establece la media prevista para analizar el } \\
\text { resultado y generar las conclusiones } \\
\text { pertinentes. }\end{array}$ \\
\hline
\end{tabular}

Elaboración propia de los autores (2018)

\section{Diseño de experimentos por factores}

Mediante el análisis factorial como lo confirma Vallejo (2013), se analiza la varianza común a todas las variables. Se parte de una matriz de correlaciones y se trata de reducir la data que da una matriz de 
correlaciones. Propiamente se opera con las correlaciones elevadas al cuadrado (r2), que expresan todo lo que hay (proporción) de varianza común entre las variables.

Long (1983) y Stapleton (1997a) demuestran que en cada casilla de la matriz de correlaciones elevadas al cuadrado se tiene la proporción de varianza común a dos ítems o variables, excepto en la diagonal, aquí aparecen "unos" (cada ítem coincide consigo mismo al 100\%). En estos "unos" se tiene la varianza que cada ítem comparte con los demás y también la que no comparte (la específica o única de cada ítem). Si se pretende examinar exclusivamente la varianza compartida se debe eliminar los "unos" de la matriz de correlaciones y poner, en su lugar, la proporción de varianza que cada ítem tiene en común con todos los demás.

Se establecen, por lo tanto, dos enfoques en el análisis factorial, a partir de lo afirmado por Nunnally (1978); Nunnally and Bernstein (1994) y Kline (1994):

1. Analizar toda la varianza, común y no común; en este caso se debe usar los "unos" de la matriz de correlaciones. El método más usual es el de análisis de Componentes Principales.

2. Analizar solamente la varianza común, y en este caso se substituyen los "unos" de la diagonal por estimaciones de la varianza que cada ítem tiene en común con los demás (y que se denominan comunalidades). Para la estimación de las comunalidades no hay un cálculo único; existen diversos procedimientos (como correlaciones múltiples de cada ítem con todos los demás, o coeficientes de fiabilidad si cada variable es un test). Estos procedimientos (en los que se substituyen los unos por las comunalidades) se denominan "análisis de factores comunes".

El análisis factorial en la arquitectura y exégesis de test, escalas y cuestionarios, desde ambos enfoques, engloban la designación genérica de análisis factorial, no obstante, es al análisis de factores comunes al que con más pertenencia se le designa el apelativo de análisis factorial; es de connotar que ambos enfoques proporcionan corolarios similares y se explican de manera equivalente.

Ahora, se debe definir el Diseño Estadístico de Experimentos (DEE), también denominado diseño experimental, como una metodología basada en útiles matemáticos y estadísticos cuyo objetivo en palabras de Ferré \& Rius (2002) es ayudar al investigador a:

1. Escoger la estrategia experimental recomendable que asiente conseguir la data buscada con el exiguo importe.

2. Valorar los corolarios experimentales logrados, certificando la máxima fiabilidad en las soluciones que se alcancen.

En los últimos años y como lo demuestra Napolitano (2010), la atención hacia el diseño experimental ha progresado claramente debido al reconocimiento que los experimentos esbozados son fundamentales para la mejora de la calidad de servicios y productos; asimismo, el gerenciamiento del control estadístico de calidad, resuelve la inspección y otras herramientas de calidad que igualmente valen para esta función, el diseño de experimentos constituye la metodología de la elección de variables e interacciones y marcos complejos. Dinámicamente, el diseño de experimentos ha progresado y mejorado en el sector público.

\section{Resultados}

Para el desarrollo estadístico multivariado se utilizaron las cuatro dimensiones (factores) definidas por el Departamento Nacional de Planeación para medir el componente de gestión del Nuevo Índice de Medición del Desempeño Municipal. Inicialmente se relacionan en la Tabla 4 las categorías, su grado positivo o negativo, los valores dicotómicos y los resultados obtenidos por las entidades territoriales por cada uno de ellos. 
TABLA 4.

Categorías del componente de gestión del nuevo índice de medición de desempeño

\begin{tabular}{llll}
\hline Dimensión & Grado & Valores & Total \\
\hline A. Movilización de & + & Alta & 114 \\
recursos & - & Baja & 987 \\
B. Ejecución de & + & Eficiente & 1019 \\
recursos & - & Ineficiente & 82 \\
C. Ordenamiento & + & Cumple & 47 \\
territorial & - & No cumple & 1054 \\
D. Gobierno abierto y & + & Organizado & 956 \\
transparencia & - & No organizado & 145 \\
\hline
\end{tabular}

Elaboración propia de los autores (2018)

Posteriormente, en la Tabla 5 se relacionan los 16 escenarios del modelo de $2^{\mathrm{k}=4}$, permitiendo determinar el orden de las variables con respecto a cada una de las posibles alternativas lógicas del modelo. El total de cada una de ellas es obtenido en la base de datos del DNP, con base en el número de veces que se generan en cada una de las combinaciones propuestas.

TABLA 5.

Organización de los valores posibles por dimensión

\begin{tabular}{|c|c|c|c|c|c|}
\hline No. & $\begin{array}{l}\text { A. Movilización de } \\
\text { recursos }\end{array}$ & $\begin{array}{l}\text { B. Ejecución de } \\
\text { recursos }\end{array}$ & $\begin{array}{l}\text { c. Ordenamiento } \\
\text { territorial }\end{array}$ & $\begin{array}{l}\text { D. Gobierno abierto y } \\
\text { transparencia. }\end{array}$ & Total \\
\hline 1 & ALTA & EFICIENTE & CUMPLE & ORGANIZADO & 71,6 \\
\hline 2 & $\mathrm{BAJA}$ & EFICIENTE & CUMPLE & ORGANIZADO & 59,5 \\
\hline 3 & ALTA & INEFICIENTE & CUMPLE & ORGANIZADO & 0 \\
\hline 4 & BAJA & INEFICIENTE & CUMPLE & ORGANIZADO & 55,8 \\
\hline 5 & ALTA & EFICIENTE & NO CUMPLE & ORGANIZADO & 61,9 \\
\hline 6 & BAJA & EFICIENTE & NO CUMPLE & ORGANIZADO & 47,9 \\
\hline 7 & ALTA & INEFICIENTE & NO CUMPLE & ORGANIZADO & 59,1 \\
\hline 8 & $\mathrm{BAJA}$ & INEFICIENTE & NO CUMPLE & ORGANIZADO & 40 \\
\hline 9 & ALTA & EFICIENTE & CUMPLE & NO ORGANIZADO & 59,7 \\
\hline 10 & $\mathrm{BAJA}$ & EFICIENTE & CUMPLE & NO ORGANIZADO & 0 \\
\hline 11 & ALTA & INEFICIENTE & CUMPLE & NO ORGANIZADO & 0 \\
\hline 12 & $\mathrm{BAJA}$ & INEFICIENTE & CUMPLE & NO ORGANIZADO & 0 \\
\hline 13 & ALTA & EFICIENTE & NO CUMPLE & NO ORGANIZADO & 50,3 \\
\hline 14 & $\mathrm{BAJA}$ & EFICIENTE & NO CUMPLE & NO ORGANIZADO & 37,3 \\
\hline 15 & ALTA & INEFICIENTE & NO CUMPLE & NO ORGANIZADO & 0 \\
\hline 16 & $\mathrm{BAJA}$ & INEFICIENTE & NO CUMPLE & NO ORGANIZADO & 30,9 \\
\hline
\end{tabular}

Elaboración propia de los autores (2018).

Una vez terminada la etapa anterior, se identifican los efectos por cada uno de los componentes del diseño de $2^{\mathrm{k}}$, seguido de la suma cuadrada de las alternativas, que conllevan a registrar los datos presentados en la Tabla 6. 
TABLA 6.

Cálculo de valores totales por cada escenario y de las diferencias por cada dimensión y combinación

\begin{tabular}{|c|c|c|c|c|c|c|c|c|c|c|c|c|c|c|c|c|c|}
\hline No. & A & B & C & D & $\mathrm{AxB}$ & $\mathrm{AxC}$ & $\mathrm{AxD}$ & $\mathrm{BxC}$ & BxD & $\mathrm{CxD}$ & $\mathrm{AxBxC}$ & AxBxD & $\mathrm{AxCxD}$ & $\mathrm{BxCxD}$ & $A x B x C x D$ & Valores & SC \\
\hline 1 & - & - & - & - & + & + & + & + & + & + & - & - & - & - & + & 71,6 & 5126,56 \\
\hline 2 & + & - & - & - & - & - & - & + & + & + & + & + & + & - & - & 59,5 & 3540,25 \\
\hline 3 & - & + & - & - & - & + & + & - & - & + & + & + & - & + & - & 0 & 0 \\
\hline 4 & + & + & - & - & + & - & - & - & - & + & - & - & + & + & + & 55,8 & 3113,64 \\
\hline 5 & - & - & + & - & + & - & + & - & + & - & + & - & + & + & - & 61,9 & 3831,61 \\
\hline 6 & + & - & + & - & - & + & - & - & + & - & - & + & - & + & + & 47,9 & 2294,41 \\
\hline 7 & - & + & + & - & - & - & + & + & - & - & - & + & + & - & + & 59,1 & 3492,81 \\
\hline 8 & + & + & + & - & + & + & - & + & - & - & + & - & - & - & - & 40 & 1600 \\
\hline 9 & - & - & - & + & + & + & - & + & - & - & - & + & + & + & - & 59,7 & 3564,09 \\
\hline 10 & + & - & - & + & - & - & + & + & - & - & + & - & - & + & + & 0 & 0 \\
\hline 11 & - & + & - & + & - & + & - & - & + & - & + & - & + & - & + & 0 & 0 \\
\hline 12 & + & + & - & + & + & - & + & - & + & - & - & + & - & - & - & 0 & 0 \\
\hline 13 & - & - & + & + & + & - & - & - & - & + & + & + & - & - & + & 50,3 & 2530,09 \\
\hline 14 & + & - & + & + & - & + & + & - & - & + & - & - & + & - & - & 37,3 & 1391,29 \\
\hline 15 & - & + & + & + & - & - & - & + & + & + & - & - & - & + & - & 0 & 0 \\
\hline \multirow[t]{2}{*}{16} & + & + & + & + & + & + & + & + & + & + & + & + & + & + & + & 30,9 & 954,81 \\
\hline & -3 & $-25,30$ & 10,10 & $-27,20$ & 20,80 & 0,10 & $-6,55$ & 8,45 & $-3,80$ & 4,60 & $-11,1$ & 5,10 & 19,30 & $-7,70$ & 7,15 & & \\
\hline
\end{tabular}

Elaboración propia de los autores (2018).

A continuación se procede a realizar la suma de cuadrado total y de cada uno de las combinaciones posibles, calculando por cada una los grados de libertad y los cuadrados medios representados en la Tabla 7.

TABLA 7.

Cálculo de valores totales por cada escenario y de las diferencias por cada dimensión y combinación

\begin{tabular}{llll}
\hline & Suma cuadrado & $\begin{array}{l}\text { Grados de } \\
\text { libertad }\end{array}$ & $\begin{array}{l}\text { Cuadrado } \\
\text { medio }\end{array}$ \\
\cline { 2 - 4 } Total & 10847,31 & 15 & 723,154 \\
A & 60,84 & 1 & 60,84 \\
B & $2.560,36$ & 1 & 2560,36 \\
C & 408,04 & 1 & 408,04 \\
D & $2.959,36$ & 1 & 2959,36 \\
AxB & $1.730,56$ & 1 & 1730,56 \\
AxC & 0,04 & 1 & 0,04 \\
AxD & 171,61 & 1 & 171,61 \\
BxC & 285,61 & 1 & 285,61 \\
BxD & 57,76 & 1 & 57,76 \\
CxD & 84,64 & 1 & 84,64 \\
AxBxC & 492,84 & 1 & 492,84 \\
AxBxD & 104,04 & 1 & 104,04 \\
AxCxD & $1.489,96$ & 1 & 1489,96 \\
BxCxD & 237,16 & 1 & 237,16 \\
AxBxCxD & 204,49 & 1 & 204,49 \\
RESIDUAL & 0 & 0 & 0 \\
\hline
\end{tabular}

Elaboración propia de los autores (2018).

A partir de estos resultados, se identifican los valores de menor valor en la suma de cuadrados para agruparlos y definir el residual (2.107,07), los grados de libertad (15 para el primero y los demás son 1 ) y el cuadrado medio (191,55). El mismo procedimiento se realiza por cada uno de los componentes y $k$ combinaciones, encontrando el $F$-ratio que se analiza con el $F$ Tabla, obtenido por la cantidad de escenarios trabajados, como se ilustra en la Tabla 8 , que además tiene resaltado aquellas opciones que cumplen con la condición inicial de ser menores de 300. 
TABLA 8.

Identificación de f ratio, f tabla y análisis de suma de cuadrados

\begin{tabular}{llllll}
\hline $\begin{array}{c}\text { Suma } \\
\text { Cuadrados }\end{array}$ & $\begin{array}{l}\text { Suma } \\
\text { cuadrado }\end{array}$ & $\begin{array}{l}\text { Grados de } \\
\text { libertad }\end{array}$ & $\begin{array}{l}\text { Cuadrado } \\
\text { medio }\end{array}$ & F-ratio & F-tablas \\
\cline { 2 - 6 } A $^{*}$ & 10847,31 & 15 & & & \\
$\mathrm{~B}$ & 60,84 & 1,00 & 60,84 & 0,32 & 4,84 \\
$\mathrm{C}$ & $2.560,36$ & 1,00 & $2.560,36$ & 13,37 & 4,84 \\
$\mathrm{D}$ & 408,04 & 1,00 & 408,04 & 2,13 & 4,84 \\
$\mathrm{AxB}$ & $2.959,36$ & 1,00 & $2.959,36$ & 15,45 & 4,84 \\
$\mathrm{AxC}$ & $1.730,56$ & 1,00 & $1.730,56$ & 9,03 & 4,84 \\
$\mathrm{AxD}$ & 0,04 & 1,00 & 0,04 & 0,00 & 4,84 \\
$\mathrm{BxC}$ & 171,61 & 1,00 & 171,61 & 0,90 & 4,84 \\
$\mathrm{BxD}$ & 285,61 & 1,00 & 285,61 & 1,49 & 4,84 \\
$\mathrm{CxD}$ & 57,76 & 1,00 & 57,76 & 0,30 & 4,84 \\
$\mathrm{AxBxC}$ & 84,64 & 1,00 & 84,64 & 0,44 & 4,84 \\
$\mathrm{AxBxD}$ & 492,84 & 1,00 & 492,84 & 2,57 & 4,84 \\
$\mathrm{AxCxD}$ & 104,04 & 1,00 & 104,04 & 0,54 & 4,84 \\
$\mathrm{BxCxD}$ & $1.489,96$ & 1,00 & $1.489,96$ & 7,78 & 4,84 \\
$\mathrm{AxBxCxD}$ & 237,16 & 1,00 & 237,16 & 1,24 & 4,84 \\
& 204,49 & 1,00 & 204,49 & 1,07 & 4,84 \\
\hline
\end{tabular}

Elaboración propia de los autores (2018).

* opciones que cumplen con la condición inicial de ser menores de 300

Al eliminar las alternativas que cumplían la condición mencionada en la Tabla 8 , se procede a calcular el residual $(2.107,07)$ con base en la suma de los cuadrados de los factores establecidos y sus grados de libertad (11) y el cuadrado medio (191,55), lo que genera una media prevista de 29,18.

TABLA 9.

Establecimiento de la media prevista

\begin{tabular}{lllll}
\hline $\begin{array}{c}\text { Suma Cuadrados } \\
\text { por factor }\end{array}$ & $\begin{array}{l}\text { Suma de cuadrado por } \\
\text { factor }\end{array}$ & Grado de libertad & Cuadrado Medio & F-ratio \\
\hline B & 2560,36 & 1 & 2560,36 & 13,36640928 \\
C & 408,04 & 1 & 408,04 & 2,130180772 \\
D & 2959,36 & 1 & 2959,36 & 15,44939656 \\
AxB & 1730,56 & 1 & 1730,56 & 9,034422207 \\
AxBxC & 492,84 & 1 & 492,84 & 4,84 \\
AxCxD & 1489,96 & 1 & 1489,96 & 4,572880825 \\
\hline
\end{tabular}

Elaboración propia de los autores (2018).

* opciones que cumplen con la condición inicial de ser menores de 300

El componente de gestión representa el 50\% del Nuevo Índice de Desempeño Municipal, el cual es un instrumento basado de los principios de la Nueva Gestión Pública que permite la medición del desarrollo de las decisiones y acciones que un Alcalde realiza en cada vigencia en Colombia, lo que se ha convertido en un referente de la administración de los recursos en los países de la región. El estudio realizado con información 2017 de las cuatro categorías con respecto a los datos reportados en el 2016, determina en el diseño de experimentos que la media prevista es de 29,18 , un valor preocupante para la gestión de la administración pública en los 1121 municipios de Colombia, que pueden estar definidos con una gestión de un poco más de la cuarta parte de un escenario ideal, esto tiene repercusiones en el desarrollo del componente de resultados, que sin gestión, difícilmente podrá estar en un estado satisfactorio. 
La realidad del resultado presentado esta alineado con la baja ejecución de los fondos de inversión púbica de los municipios, como por ejemplo se visualiza en el Sistema General de Regalías, que tiene una ejecución bianual que no supera el margen del $48 \%$ del total disponible. Es así, que con estos resultados el objetivo principal de la administración pública de generar el máximo bienestar posible con los recursos del Estado no se cumple a cabalidad en el territorio del país, conllevando a altos niveles de desigualdad económica, de desarrollo social y de necesidades básicas insatisfechas.

Se recurre al diseño experimental como el medio para analizar componentes de gestión del nuevo índice de medición municipal en Colombia; debido a que, es un instrumento de los métodos multivariados que tienen un potencial importante para conceptualizar y diseñar estrategias que optimicen no solo procesos industriales, si no procesos a nivel de servicios; por ello es que los diseños factoriales completos son la estrategia experimental recomendable para experimentar simultáneamente el resultado de diferentes factores sobre la respuesta y sus interacciones al poder identificar qué variables influyen en una reacción, para luego poder optimizarlas hasta alcanzar el rendimiento deseado y para decidir qué se debe ajustar.

El estado colombiano, en la actualidad, cuenta con diferentes sistemas de información abiertos que permiten a los investigadores y centros de pensamiento reflexionar sobre estos datos, para crear y gestionar un conocimiento que promueva la calidad de vida de los colombianos.

De acuerdo con el análisis factorial, las dimensiones que los alcaldes en Colombia deben priorizar dentro de sus modelos de gestión son la ejecución de recursos, el ordenamiento territorial y el gobierno abierto y transparente. En cuanto a las combinaciones más importantes por desarrollar en los municipios del país y mejorar los niveles de la gestión pública son en orden: 1) la movilización de recursos con ejecución de recursos, 2) los dos anteriores con el ordenamiento territorial y 3) el desarrollar los cuatro simultáneamente.

Los datos son representaciones de la realidad, que toman valor cuando se convierten en sistemas de información que orienten la toma de decisiones. Para la administración del Estado el índice de Medición del Desempeño Municipal permite entender la realidad de los mecanismos de operacionalización y la efectividad de la consecución de los resultados mínimos requeridos en el territorio. Por ende, es fundamental que los administradores de lo público reflexionen, lideren y evalúen las características, los medios y las estrategias para fortalecer el componente de gestión, que debe estar al cien por ciento de su posibilidad, entre mayor sea, mayor convivencia, riqueza y prosperidad; los territorios se beneficiarían y generarían un mejor impacto en la gestión a nivel nacional.

Los resultados de esta investigación aportan avances claves que demuestran como la academia y la investigación pueden ser un soporte estratégico para el fortalecimiento de la administración pública en Colombia y en la región. Se invita a continuar con este tipo de estudios cuantitativos en el análisis de los sistemas de información utilizados para la toma de decisiones. Entender las dinámicas, evaluar el impacto y determinar planes estratégicos basados en los componentes y dimensiones del indicador de medición de desempeño municipal generara mayor conocimiento, que pueda ser replicado a nivel departamental, nacional e internacional.

\section{REFERENCIAS BIBLIOGRÁFICAS}

Agüero, J. O. (2007). Teoría de la administración: un campo fragmentado y multifacético. Revista Científica" Visión de Futuro", 7(1).

Albarracin Rodriguez, A. P. (2012). Análisis factorial confirmatorio del cuestionario de análisis clínico (CAQ) en una muestra de jóvenes de bucaramanga. Psychologia : Avances De La Disciplina, 6(2), 35-43. Retrieved from https ://bdbiblioteca.universidadean.edu.co:2083/docview/1793563859?accountid=34925

Avella, B., \& Oliva, M. (2010). Comparación del análisis factorial múltiple (AFM) y del análisis en componentes principales para datos cualitativos (Prinqual), en la construcción de índices/Comparison between multiple factor 
analysis (MFA) and principal component analysis for qualitative data (Prinqual) methods for derivation of indices (Doctoral dissertation, Universidad Nacional de Colombia)

Bertalanffy, V. L. T. G. D. (1992). Sistemas. São Paulo.

Bueno, J. G. S. (1993). Educação especial brasileira: integração/segregação do aluno diferente. EDUC-Editora da PUC-SP.

Burke, R. J., Jeng, W., Koyuncu, M. \& Fiksenbau, L. (2011). Work motivations, satisfaction and well-being among hotel managers in China: Passion versus addiction. Interdisciplinary Journal of Research in Business, 1, 21-34.

Burt, C. (1949). La estructura de la mente; Una revisión de los resultados del análisis factorial. Revista británica de psicología educativa .

Cassar, V. (2001). Violating psychological contract terms amongst Maltese public service employees: occurrence and relationships. Journal of Managerial Psychology, 16 (3), 194-208

CLAD, C. C. (1999). UNA NUEVA GESTIÓN PÚBLICA PARA AMÉRICA LATINA. Economía Y Sociedad, 4(09). Recuperado a partir de https://www.revistas.una.ac.cr/index.php/economia/article/view/1577

Clarkson, M. (1995). A stakeholder framework for analyzing and evaluating corporate social performance. Academy of Management Review, 20 (1), 92-117.

Chiaburu, D. S., Peng, A. C., Oh, I.-S., Banks, G. C. y Lomeli, L. C. (2013). Antecedents and consequences of employee organizational cynicism: A meta-analysis. Journal of Vocational Behavior, 83, 181-197. http://dx.doi.org/10.1 016/jjvb.2013.03.007

Carretero, M. (2017). Cambio conceptual y enseñanza de la historia. Tarbiya, Revista de Investigación e Innovación Educativa, (26).

Claver, E., Llopis, J., Gascó, J. L., Molina, H., \& Conca, F. J. (1999). Public administration: From bureaucratic culture to citizen-oriented culture. International journal of public sector management, 12(5), 455-464.

Courtney, J. F. (2001). Decision making and knowledge management in inquiring organizations: toward a new decision-making paradigm for DSS. Decision support systems, 31(1), 17-38.

Chen, H. (2006). Flow on the net-detecting web user's positive affects and their flow states. Computers in Human Behavior, Vol. 22, pp. 221- 233

Christensen, T., \& Lægreid, P. (2010). Increased complexity in public organizations-the challenges of combining NPM and post-NPM. In Governance of public sector organizations (pp. 255-275). Palgrave Macmillan, London.

Departamento Nacional de Planeación (2017) Guía orientaciones para realizar la medición del desempeño integral. Guía para oficinas departamentales de Planeación. Bogotá

Departamento Nacional de Planeación (2016) Nueva Medición de Desempeño Institucional MDM. Oficina de Planeación. Bogotá

Departamento Nacional de Planeación (2017) Información consolidada de municipios de Colombia. Bogotá

DuBrin, A. J. (2000). Fundamentos de administración (No. 658 D8Y 2000).

Dunleavy, P., \& Hood, C. (1994). From old public administration to new public management. Public money \& management, 14(3), 9-16.

Drucker, D. C. (1966). Continuum theory of plasticity on macroscale and microscale. Journal of Materials, 1(4), 873.

Fayol, H. (1918). Administration industrielle et générale (Vol. 2). Dunod et Pinat.

Ferrando, P. J., \& Anguiano-Carrasco, C. (2010). El análisis factorial como técnica de investigación en psicología. Papeles del psicólogo, 31(1), 18-33.

Ferré, J., \& Rius, F. X. (2002). Introducción al diseño estadístico de experimentos. TECNICAS DE LABORATORIO-BARCELONA-, 648-653.

Fisher, L. \& Locke, E. (1992). The California family health project: V. Family problem solving and adult health. Family process, 31(4), 421-431.

Galton, F. (1883). Mental imagery. 
García, M. I. B., Enrique, V. I. L. A., \& Tello, F. P. H. (2011). Introducción básica al análisis factorial. Editorial UNED. Garmendia, M. L. (2007). Análisis factorial: una aplicación en el cuestionario de salud general de Goldberg, versión de 12 preguntas. Revista chilena de salud pública, 11(2), 57-65.

Gardner, D. G., Huang, G.-H., Niu, X., Pierce, J. L. y Lee, C. (2015). Organization-based self-esteem, psychological contract fulfillment, and perceived employment opportunities: A test of self-regulatory theory. Human Resource Management, 54(6), 933-953. http://dx.doi.org/10.1002/hrm.21648

Gibson, J. K. (1997). The end of capitalism (as we knew it): A feminist critique of political economy. Capital \& Class, 21(2), 186-188.

González M, Ibáñez I. (2001). Cuestionario de Salud General (GHQ-12): comparación de dos modelos factoriales. Psquiatriacom.

Guaderrama, A. I. M., Arroyo, J. C., \& Flores, G. R. (2017). Efecto de la violación del contrato psicológico y el agotamiento emocional sobre el cinismo del empleado. Estudios Gerenciales, 33(143), 124-131. doi:http://bd biblioteca.universidadean.edu.co:2156

Gulick, L. (1937). Notes on the Theory of Organization. Classics of organization theory, 3.

Jamali, D. and Neville, B. (2011). Convergence versus divergence of CSR in developing countries:

Johnson, J. L., \& O'Leary-Kelly, A. M. (2003). The effects of psychological contract breach and organizational cynicism: Not all social exchange violations are created equal. Journal of Organizational Behavior, 24, 627-647.

Long, J. Scott (1983), Confirmatory Factor Analysis: A Preface to LISREL, Paper Series on Quantitative Applications in the Social Sciences, Newbury Park \& London, Sage

Luhmann, N. (1996). Protest: systemtheorie und soziale Bewegungen (Vol. 1256). Suhrkamp.

Manrique, H. (2006). The changing psychological contract: Challenges and implications for HRM, organizations and employees. In R., Wiesner, B. \& Millett (eds.) Human resource management: Challenges \& future direction (pp. 8710). Australia: John Wiley \& Sons.

Miller, E. J., \& Rice, A. K. (2013). Systems of organization: The control of task and sentient boundaries. Routledge.

Mitchell, R., Agle, B. y Wood, D. (1997). Toward a theory of stakeholderidentifi cation and salience: defi ning the principle of who and what really counts. Academy of Management Review, 22, 853-886

Morrison D. (1990). Multivariate Statistical Methods: New York: Mac Graw-Hill.

Napolitano, H. (2010). Diseño de experimentos. Journal Article.

Naus, A.J.A.M. (2007). Organizational cynicism: on the nature, antecedents, and consequences of employee cynicism toward the employing organization. Unpublished Doctoral thesis. University Maastricht. Consultado Noviembre 14, 2010 en http://arnounimaasnl/showcgif.

Nunnally, Jum C. (1978). Psychometric Theory. New York: McGraw-Hill. 45.

Nunnally, Jum C. and Bernstein, Ira H. (1994). Psychometric Theory, third edition. New York: McGraw-Hill.

Morgan, D. P., (1991). Surface-wave devices for signal processing (Vol. 1991). Amsterdam: elsevier.

Nisbet, R. A. (1948). The Politics of Social Pluralism: Some Reflections on Lamennais. The Journal of Politics, 10(4), 764-786.

Organización para el Desarrollo Económico- OCDE.(2010). PISA 2009 Results.

Pollitt, C., \& Bouckaert, G. (2004). Public management reform: A comparative analysis. Oxford University Press, USA.

Prats, J. (2004). Gobernabilidad para el desarrollo. Propuesta de un marco conceptual y analítico. Democracia con desigualdad, 3-33.

Sánchez, G. J. J. (Ed.). (2009). Derecho mercantil (Vol. 1). Grupo Planeta (GBS).

Seligman, M. E., Steen, T. A., Park, N., \& Peterson, C. (2005). Positive psychology progress: empirical validation of interventions. American psychologist, 60(5), 410.

Sen, A. (2017). Collective choice and social welfare: Expanded edition. Penguin UK. 
Iván Rodrigo Vargas Ramírez, et aL. AnÁlisis factorial del componente de gestión del nUevo índice ...

Sinclair, A. (1995). The chameleon of accountability: forms and discourses. Accounting, organizations and Society, 20(2-3), 219-237.

Spearman, Ch. (1904). General intelligence; objectively determined and measured. American Journal of Psychology, $115,201292$.

Spearman, C. (1904). Inteligencia general determinada y medida objetivamente. Am J Psich, 5, 201-293.

Stapleton, Connie D. (1997a). Basic Concepts in Exploratory Factor Analysis (EFA) as a Tool to Evaluate Score Validity: A Right-Brained Approach. Paper presented at the anual meeting of the Southwest Educational Research Association, Austin, reproducido en How To Series: En http://mirror.eschina.bnu.edu.cn/Mirror1/ accesseric/ericae.net/ft/tamu/Efa.html

Thomson, R. M. (1951). Studies on salt-water and fresh-water Anopheles gambiae on the East African coast. Bulletin of entomological research, 41(3), 487-502.

Topa, G., \& Palací, F. J. (2004). ¿RUPTURA O CUMPLIMIENTO DEL CONTRATO PSICOLÓGICO? UNA REVISIÓN META-ANALÍTICA DE LA INVESTIGACIÓN EMPÍRICA/¿PSYCHOLOGICAL CONTRACT, BREACH OR FULFILMENT? A META-ANALYTIC REVIEW OF EMPIRIC RESEARCH. Acción psicológica, 3(3), 155.

Taylor, F. W. (1911). Administración científica. Barcelona: Ediciones Orbis.

Toffler, A. (1997). Foreword: The new intangibles. In Athena’s Camp.

Thompson, J. L. (2001). Strategic management. Thompson Learning.

Turnley, W. H., Bolino, M. C., Lester, S. W., \& Bloodgood, J. M. (2003). The impact of psychological contract fulfillment on the performance of in-role and organizational citizenship behaviors. Journal of management, 29(2), 187-206.

Thurstone, L. L. (1931). The measurement of social attitudes. The journal of abnormal and social psychology, 26(3), 249.

Thurstone, LL (1935). Los vectores de la mente: análisis de factores múltiples para el aislamiento de rasgos primarios.

Thurstone, L. L. (1947). Multiple factor analysis, Chicago, IL: University of Chicago Press.

Urwick, L. F. (1922). The manager's span of control. Harvard Business Review, 34(3).

Vallejo, P. M. (2013). El análisis factorial en la construcción e interpretación de tests, escalas y cuestionarios. Madrid: Universidad Pontificia Comillas.

Vargas, I., R. (2019) La gestión de la felicidad y su relación con la administración pública en la gobernaciones de Colombia.Ide@as CONCYTEG. México

Vidal, G. H. (2016). Identificación y evaluación de los factores de riesgo psicosocial. XXXII Congreso de Seguridad Integral, Higiene y Medicina del Trabajo. Consejo Colombiano de Seguridad

Villegas, M. G. (2007). La teoría de los stakeholders y la emisión de información para todos los interesados: ¿discurso o transparencia informativa? Contaduría Universidad De Antioquia, (49), 95-102. Retrieved from https://bdb iblioteca.universidadean.edu.co:2083/docview/198677694?accountid=34925

Weber, M. (1922). La dominación no legítima (tipología de las ciudades). Economía y sociedad, 938-1046.

\section{Notas}

[1] La data compartida por cada una de las instituciones territoriales del país en las variables movilización de recursos, ejecución de recursos y ordenamiento territorial se realiza a través del Formato Único Territorial (FUT) a través del sistema de información del Consolidador de Hacienda y Crédito Público (CHIP), mientras que la variable de gobierno abierto y transparente es remitida por la plataforma GESTIONWEB del Departamento Nacional de Planeación.

La Universidad de Cuenca en Ecuador, conserva los derechos patrimoniales (copyright) de las obras publicadas, y favorece y permite la reutilización de las mismas bajo la licencia Creative Commons AtribuciónNoComercial-CompartirIgual 4.0 Internacional (CC BY-NC-SA 4.0), por lo cual se pueden copiar, usar, 
difundir, transmitir y exponer públicamente, siempre que: a. Se cite la autoría y fuente original de su publicación (revista, editorial, URL y DOI de la obra). b. No se usen para fines comerciales u onerosos. c. Se mencione la existencia y especificaciones de esta licencia de uso.

CC BY-NC-SA 